\title{
INVERSE LIMITS AND HOMOGENEITY $\left({ }^{1}\right)$
}

\author{
BY \\ RICHARD M. SCHORI
}

1. Introduction. In 1958 Jack Segal [4] announced some sufficient conditions on an inverse limit sequence for the limit space to be homogeneous. However, this result was not verified and in $1965 \mathrm{McC}$ Cord [3] proved a weaker version of the theorem. In this paper we prove a partial converse to McCord's theorem which enables us to establish a counterexample to Segal's original theorem.

Segal announced: If, in an inverse limit sequence of topological spaces, the coordinate spaces are path-connected, locally path-connected, and homogeneous, and the bonding maps are covering maps, then the inverse limit space is homogeneous. McCord proved that if $\left(X_{n}, \pi_{m}^{n}\right)$ is an inverse sequence of manifolds and each $\pi_{1}^{n}: X_{n} \rightarrow X_{1}$ is a regular covering map, then the limit space is homogeneous. Theorem 4 of this paper says roughly that if the bonding maps are badly nonregular, then the limit space is not homogeneous. An inverse limit of compact 2 -manifolds is then constructed satisfying the conditions of Theorem 4. In [3, p. 208] McCord has conjectured the existence of such an example. Thus, we see that in spirit Segal's theorem was right, but curiously it needed the somewhat natural condition of regularity on the covering maps to force homogeneity on the limit space. The author thanks the referee for many suggestions and in particular for the formulation of Theorem 4 .

2. Preliminaries. If $X$ and $Y$ are connected and locally path-connected spaces, then $p: X \rightarrow Y$ is a covering map if (1) $p$ is a map from $X$ onto $Y$ and (2) for each point $y$ in $Y$ there exists a connected open set $U$ containing $y$ such that each component of $p^{-1}(U)$ is open in $X$ and is mapped homeomorphically onto $U$ by $p$. The set $U$ is called a canonical neighborhood with respect to the covering map $p$. See [2] for the definition of a covering map to be regular. If $p: X \rightarrow Y$ is a covering map, $u: I \rightarrow Y$ is a path in $Y$, and $v: I \rightarrow X$ is a path in $X$, then we say that $u$ lifts to $v$ iff $p \circ v=u$. If $X$ is a space, $u: I \rightarrow X$ is a path, and $x \in X$, then $u$ is a loop on $x$ iff $u(0)=u(1)=x$. A necessary and sufficient condition for a covering map $p$ to be regular is that if $w$ is any loop in $Y$ on $y$ that lifts to a loop for some point

Presented to the Society, April 10, 1965; received by the editors August 10, 1965 and, in revised form, March 18, 1966.

(1) This paper is part of the author's doctoral dissertation at the University of Iowa, prepared under the supervision of Professor Steve Armentrout while the author was supported on an NSF Cooperative Fellowship. 
of $p^{-1}(y)$, then $w$ lifts to a loop for all points of $p^{-1}(y)$. The map $f_{1}^{2}$ of Figure 3 is an example of a nonregular covering map.

The symbol $\left(M_{i}, f_{i-1}^{i}\right)$ stands for an inverse limit sequence where if $i \geqq 1$, $M_{i}$ is a space, if $i>1, f_{i-1}^{i}$ is a map from $M_{i}$ into $M_{i-1}$, if $1 \leqq i \leqq j$, then $f_{i}^{j}=f_{i}^{i-1} \circ \cdots \circ f_{j-1}^{j}$, and if $M$ is the limit space then $f_{i}: M \rightarrow M_{i}$ is the natural projection.

3. Results. The first two theorems are probably known, but are not to my knowledge in the literature.

THEOREM 1. Let $M$ be the limit of an inverse sequence $\left(M_{i}, f_{i-1}^{i}\right)$ where each bonding map is a covering map. Two points $x=\left(x_{i}\right)$ and $y=\left(y_{i}\right)$ of $M$ are in the same path component of $M$ if and only if there exists a path

$$
v_{1}:(I, 0,1) \rightarrow\left(M_{1}, x_{1}, y_{1}\right)
$$

such that for each $i>1, v_{1}$ lifts to a path $v_{i}:(I, 0,1) \rightarrow\left(M_{i}, x_{i}, y_{i}\right)$.

Proof. Assume $x$ and $y$ are in the same path-component of $M$ and let $v:(I, 0,1) \rightarrow(M, x, y)$. If $i \geqq 1$, define $v_{i}:(I, 0,1) \rightarrow\left(M_{i}, x_{i}, y_{i}\right)$ such that $v_{i}=f_{i} \circ v$, and thus the collection $v_{1}, v_{2}, \cdots$ satisfies the condition.

Assume now that the condition holds and define $v: I \rightarrow M$ such that $v(t)=\left(v_{i}(t)\right)$. Then $v$ is a path in $M$ from $x$ to $y$ and hence $x$ and $y$ are in the same pathcomponent of $M$.

Since a 1-fold covering map is a homeomorphism, from now on let us assume that all covering maps are at least 2-fold.

THEOREM 2. If $\left(M_{i}, f_{i-1}^{i}\right)$ is an inverse sequence of compact manifolds, where each bonding map is a covering map, then the limit space $M$ has $c$ path components. (In [3] McCord has shown this result for the case when each $f_{1}^{i}$ is a regular covering map.)

Proof. If $x_{1} \in M_{1}$, the set $f_{1}^{-1}\left(x_{1}\right)$ is a Cantor set and thus has cardinality $c$. Let $K$ be any path-component of $M$ that intersects $f_{1}^{-1}\left(x_{1}\right)$. We will show that $K \cap f_{1}^{-1}\left(x_{1}\right)$ is countable. Let $x \in K \cap f_{1}^{-1}\left(x_{1}\right)$ and let $\pi\left(M_{1}, x_{1}\right)$ be the fundamental group of $M_{1}$ with base point $x_{1}$. If $\alpha \in \pi\left(M_{1}, x_{1}\right)$, then $\alpha=\left[v_{1}\right]$ where $v_{1}$ is a loop in $M_{1}$ on $x_{1}$. If $i>1$, lift $v_{1}$ to $v_{i}:(I, 0) \rightarrow\left(M_{i}, x_{i}\right)$ the path in $M_{i}$ starting at $x_{i}$. Thus $\left(v_{1}(1)\right)$ is a point of $f_{1}^{-1}\left(x_{1}\right)$ and by Theorem $1\left(v_{i}(1)\right) \in K$. Define $\phi: \pi\left(M_{1}, x_{1}\right) \rightarrow K \cap f_{1}^{-1}\left(x_{1}\right)$ such that $\phi(\alpha)=\left(v_{i}(1)\right)$. By [2, Theorem 6.5.9] $\phi$ is well defined and by Theorem $1 \phi$ is onto. Hence, since $\pi\left(M_{1}, x_{1}\right)$ is countable, $K \cap f_{1}^{-1}\left(x_{1}\right)$ is countable. Thus, since the cardinality of $f_{1}^{-1}\left(x_{1}\right)$ is $c$, since each path-component of $M$ intersects at most a countable subset of $f_{1}^{-1}\left(x_{1}\right)$, and since $a \cdot \aleph_{0}=a_{0}$ for every infinite cardinal $a, M$ has $c$ path-components.

For a compact metric space $(M, d)$, let $\operatorname{Map}(M, M)$ be the separable space of all maps of $M$ into $M$ with metric $d(g, h)=\sup \{d(g(x), h(x)): x \in M\}$. 
THEOREM 3. Let $M$ be the limit of an inverse sequence $\left(M_{i}, f_{i-1}^{i}\right)$ of compact manifolds where each bonding map is a covering map. Suppose $M$ is homogeneous. Let $\varepsilon>0$ and $r \in M$ be given. Then there exists a homeomorphism $h: M \rightarrow M$ such that $d(h, i d)<\varepsilon$ and $h(r) \neq r$, but $f_{1} h(r)=f_{1}(r)$.

Proof. By Theorem 2 let $S$ be an uncountable subset of $M$ such that distinct members of $S$ belong to different path components of $M$. By homogeneity of $M$, choose for each $s$ in $S$ a homeomorphism

$$
h_{s}:(M, s) \rightarrow(M, r)
$$

Now $\left\{h_{s}: s \in S\right\}$ is an uncountable subset of the separable metric space $\operatorname{Map}(M, M)$. Hence there are distinct points $s^{0}, s^{1}, s^{2}, \cdots$ in $S$, so that, setting $h_{i}=h_{s}$, the sequence $h_{1}, h_{2}, \cdots$ converges to $h_{0}$. Define $g_{i}: M \rightarrow M$ such that $g_{i}=h_{i} h_{0}^{-1}$. Since $s^{0}, s^{1}, s^{2}, \cdots$ all belong to different path-components of $M, r=h_{0}\left(s^{0}\right)$, $h_{0}\left(s^{1}\right), h_{0}\left(s^{2}\right), \cdots$ also belong to different path components and $g_{i}\left(h_{0}\left(s^{i}\right)\right)=r$.

Let $U(r, \varepsilon / 4)$ be the $\varepsilon / 4$-sphere about $r$ in $M$. There exists an integer $n$ and open cell $U_{n}$ in $M$ containing $r_{n}$ such that $f_{n}^{-1}\left(U_{n}\right) \subset U(r, \varepsilon / 4)$. Since the $h_{0}\left(s^{i}\right)$ converge to $r$ and the $g_{i}$ converge uniformly to the identity, there exists an integer $k$ such that $h_{0}\left(s^{k}\right) \in f_{n}^{-1}\left(U_{n}\right)$ and $d\left(g_{k}^{-1}, i d\right)<\varepsilon / 2$. We have $h_{0}\left(s^{k}\right)_{n}$ and $r_{n}$ in $U_{n}$ and thus we may let $u_{n}$ be a homeomorphism of $M_{n}$ onto itself that is the identity on the complement of $U_{n}$ and takes $h_{0}\left(s^{k}\right)_{n}$ to $r_{n}$. For each $i>n$, the set $\left(f_{n}^{i}\right)^{-1}\left(U_{n}\right)$ is the disjoint union of open sets each mapped homeomorphically onto $U_{n}$ by $f_{n}^{i}$. Thus $u_{n}$ lifts to a homeomorphism $u_{i}: M_{i} \rightarrow M_{i}$ and hence induces a homeomorphism $u: M \rightarrow M$.

Since $u$ is the identity on the complement of $f_{n}^{-1}\left(U_{n}\right)$ and $\operatorname{diam}\left(f_{n}^{-1}\left(U_{n}\right)\right)<\varepsilon / 2$, we have $d(u, i d)<\varepsilon / 2$. Now let $v_{n}: I \rightarrow U_{n}$ be a path in $U_{n}$ from $h_{0}\left(s^{k}\right)_{n}$ to $r_{n}$. If $j>n$, lift $v_{n}$ to $v_{j}$, the path in $M_{j}$ starting at $h_{0}\left(s^{k}\right)_{j}$. Let $t$ be the point of $f_{n}^{-1}\left(r_{n}\right)$ determined by the $v_{j}(1)$. Hence $u\left(h_{0}\left(s^{k}\right)\right)=t$ and by Theorem $1, t$ is in the same path component as $h_{0}\left(s^{k}\right)$ and thus $t \neq r$. Define $h$ such that $h=u \circ g_{k}^{-1}$. Since $d\left(g_{k}^{-1}, i d\right)<\varepsilon / 2$ we have $d(h, i d)<\varepsilon$ and $h(r)=t \neq r$. Also, since $t_{n}=r_{n}$ we have $f_{1}(h(r))=! f_{1}(r)=r_{1}$.

In the following, let us call paths $\alpha_{0}, \alpha_{1}: I \rightarrow X$ homotopic, denoted $\alpha_{0} \simeq \alpha_{1}$, only when there exists a homotopy $\alpha_{t}: I \rightarrow X$ from $\alpha_{0}$ to $\alpha_{1}$ leaving end points fixed. Thus $\alpha_{s}(0)=\alpha_{t}(0)$ and $\alpha_{s}(1)=\alpha_{t}(1)$ for all $s, t \in I$.

LEMMA 1. Let $M$ be a compact manifold with metric $d$. There exists an $\varepsilon>0$ such that if $\alpha$ and $\beta$ are paths in $M$ with $\alpha(0)=\beta(0)$ and $\alpha(1)=\beta(1)$ and $d(\alpha, \beta)<\varepsilon$, then $\alpha \simeq \beta$.

The proof is an immediate corollary to [2, Theorem 1.8 .5$]$.

THEOREM 4. Let $\left(M_{i}, f_{i-1}^{i}\right)$ be an inverse sequence of compact manifolds where each bonding map is a covering map. Suppose there exists a point $r \in M$ 
with the following property. If $k>1$ and $s_{k}$ is any point of $M_{k}$ distinct from $r_{k}$ where $f_{k-1}^{k}\left(s_{k}\right)=r_{k-1}$, then there exists a loop $\alpha: I \rightarrow M$ on $r$ and a path $\alpha_{k}^{\prime}: I \rightarrow M_{k}$ with initial point $s_{k}$ such that $f_{1}^{k} \alpha_{k}^{\prime}=\alpha_{1}$, but $\alpha_{k}^{\prime}$ is not a loop. Then $M$ is not homogeneous.

Proof. Suppose $M$ is homogeneous. Choose metrics $d_{1}$ on $M_{1}$ and $d$ on $M$ such that if $x, y \in M$, then $d_{1}\left(x_{1}, y_{1}\right) \leqq d(x, y)$. This is possible by the work of Fort and Segal in [1]. Choose $\varepsilon>0$ for $\left(M_{1}, d_{1}\right)$ as in Lemma 1 . Let $r$ be as above. By Theorem 3 we can choose a homeomorphism $h: M \rightarrow M$ such that $s=h(r) \neq r$, $s_{1}=r_{1}$, and $d(h, i d)<\varepsilon$. Since $r \neq s$ there exists $k>1$ such that $r_{k} \neq s_{k}$ but $r_{k-1}=s_{k-1}$. Let $\alpha$ and $\alpha_{k}^{\prime}$ be as above. If $\beta: I \rightarrow M$ is the path $\beta=h \alpha$, then $d_{1}\left(\alpha_{1}, \beta_{1}\right)<\varepsilon$ and hence by Lemma $1, \alpha_{1} \simeq \beta_{1}$. Now both $\alpha_{k}^{\prime}$ and $\beta_{k}$ are paths with initial point $s_{k}$ and $f_{1}^{k} \alpha_{k}^{\prime}=\alpha_{1} \simeq \beta_{1}=f_{1}^{k} \beta_{k}$. Hence, by [2, Theorem 6.9.5], $\alpha_{k}^{\prime}(1)=\beta_{k}(1)$. However, $\beta_{k}(1)=f_{k} h \alpha(1)=f_{k}(s)=s_{k}$. Thus $\alpha_{k}^{\prime}$ is indeed a loop (on $s_{k}$ ). This contradiction completes the proof.

We shall construct an inverse sequence $\left(M_{i}, f_{i-1}^{i}\right)$ and then verify that it satisfies the hypothesis of Theorem 4.

EXAMPLE. Let $M_{1}$ be a double torus or a sphere with two handles. If $n$ is a positive integer, by an $n$-handle we mean a torus with $n$ mutually disjoint open disks punched out. Thus $M_{1}$ is the union of two 1-handles, $H_{1}$ and $H_{1}^{\prime}$ whose intersection, $B$, is equal to the boundary of each of them. Let $C_{1}^{\prime \prime}$ and $D_{1}^{\prime \prime}$ be meridianal simple closed curves on $H_{1}$ and $H_{1}^{\prime}$, respectively. See Figure 1.

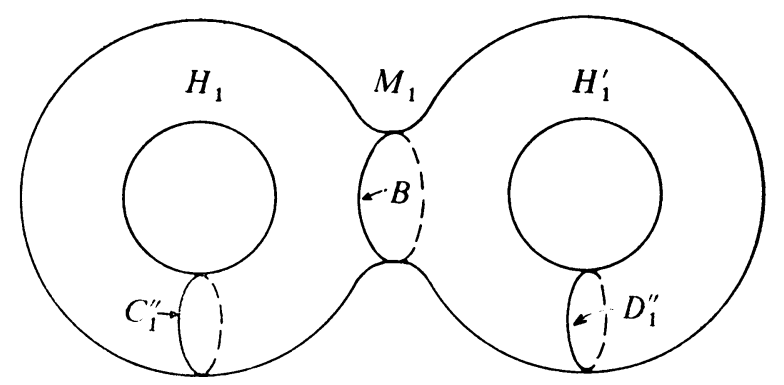

Figure 1

Suppose $k \geqq 1$ and assume that (1) the (sphere with $3^{k-1}+1$ handles), $M_{k}$, has been defined, (2) two $2^{k-1}$-handles, $H_{k}$ and $H_{k}^{\prime}$, on $M_{k}$ have been distinguished, and (3) $C_{k}^{\prime \prime}$ and $D_{k}^{\prime \prime}$ are simple closed curves on $H_{k}$ and $H_{k}^{\prime}$, respectively. Let $M_{k}^{*}=M_{k} \backslash\left(C_{k}^{\prime \prime} \cup D_{k}^{\prime \prime}\right)$ and let $h_{k}^{*}: M_{k}^{*} \rightarrow E^{3}$ be a homeomorphism that pulls the cut handles apart as shown in Figure 2 for the case when $k=1$. Let $M_{k}^{\prime}=\mathrm{Cl}\left[h_{k}^{*}\left(M_{k}^{*}\right)\right]$ and thus, the homeomorphism $h_{k}^{*-1}: h_{k}^{*}\left(M_{k}^{*}\right) \rightarrow M_{k}^{*}$ has a unique extension to a continuous function $h_{k}$ from $M_{k}^{\prime}$ onto $M_{k}$. Let $C_{k}$ and $C_{k}^{\prime}$ $\left(D_{k}\right.$ and $D_{k}^{\prime}$ ) denote the two simple closed curves that are boundary components of $M_{k}^{\prime}$ and that map onto $C_{k}^{\prime \prime}\left(D_{k}^{\prime \prime}\right)$ under $h_{k}$. 


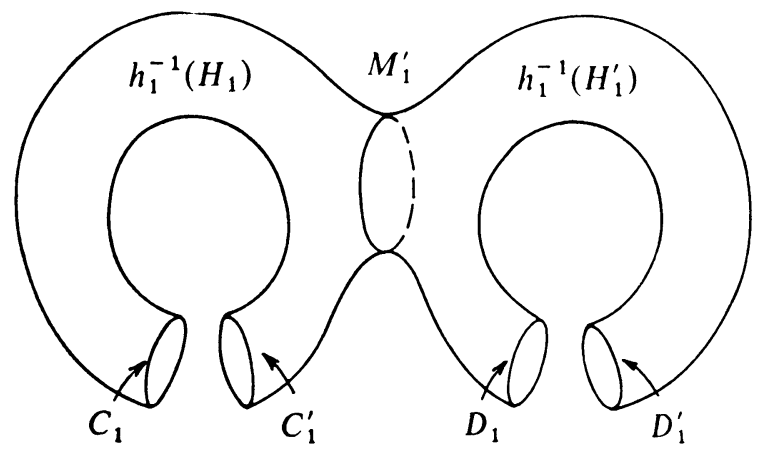

FIGURE 2

Let $\{1,2,3\}$ be a discrete space on three symbols and if $i=1,2$, or 3 , let $M_{k i}=M^{\prime} \times i, C_{k i}=C_{k} \times i, C_{k i}^{\prime}=C_{k}^{\prime} \times i, D_{k i}=D_{k} \times i, D_{k i}^{\prime}=D_{k}^{\prime} \times i, H_{k i}$ $=h_{k}^{-1}\left(H_{k}\right) \times i$, and $H_{k i}^{\prime}=h_{k}^{-1}\left(H_{k}^{\prime}\right) \times i$. Thus, if $i=1,2$, or $3, h_{k}$ induces the map $h_{k i}$ from $M_{k i}$ onto $M_{k}$ such that if $(x, i) \in M_{k i}$, then $h_{k i}(x, i)=h_{k}(x)$. Define $u_{k 1}: C_{k 1} \rightarrow C_{k 1}^{\prime}$ such that if $(x, 1) \in C_{k 1}$, then $u_{k 1}(x, 1)=h_{k 1}^{-1} h_{k}(x) \cap C_{k 1}^{\prime}$, $u_{k 2}: C_{k 2} \rightarrow C_{k 3}$ such that if $(x, 2) \in C_{k 2}$, then $u_{k 2}(x, 2)=h_{k 3}^{-1} h_{k}(x) \cap C_{k 3}^{\prime}$, and $u_{k 3}: C_{k 3} \rightarrow C^{\prime}{ }_{2}$ such that if $(x, 3) \in C_{k 3}$, then $u_{k 3}(x, 3)=h_{k 2}^{-1} h_{k}(x) \cap C_{k 2}^{\prime}$. Define $v_{k 1}: D_{k 1} \rightarrow D_{k 2}^{\prime}, v_{k 2}: D_{k 2} \rightarrow D_{k 1}^{\prime}$, and $v_{k 3}: D_{k 3} \rightarrow D_{k 3}^{\prime}$ similarly. Let $A_{k}=\bigcup_{i=1}^{3}\left(C_{k i} \cup D_{k i}\right), B_{k}=\bigcup_{i=1}^{3}\left(C_{k i}^{\prime} \cup D_{k i}^{\prime}\right)$, and $u_{k}: A_{k} \rightarrow B_{k}$ such that $u_{k} \mid C_{k i}=u_{k i}$ and $u_{k} \mid D_{k i}=v_{k i}$.

Let $M_{k+1}=\left(\bigcup_{i=1}^{3} M_{k i}\right) / u_{k}$, the identification space where each point of $A_{k}$ is identified with its image under $u_{k}$ and whose topology is the identification topology. $M_{k+1}$ is a sphere with $3^{k}+1$-handles. If $(x, i) \in M_{k i}$, let $[x, i]$ be the point of $M_{k+1}$ which is the equivalence class containing $(x, i)$. Define $f_{k}^{k+1}([x, i])$ $=h_{k}(x)$. This function is well defined and is a three fold covering map of $M_{k}$. Define $H_{k+1}$ and $H_{k+1}^{\prime}$ to be the $2^{k}$-handles $\left[H_{k 2} \cup H_{k 3}\right]$ and $\left[H_{k 1}^{\prime} \cup H_{k 2}^{\prime}\right.$, respectively. Let $C_{k+1}^{\prime \prime}$ and $D_{k+1}^{\prime \prime}$ be the simple closed curves $\left[C_{k 2}\right]=\left[C_{k 3}^{\prime}\right]$ and $\left[D_{k 1}\right]=\left[D_{k 2}^{\prime}\right]$ on $H_{k+1}$ and $H_{k+1}^{\prime}$, respectively. Note that $C_{k+1}^{\prime \prime}$ connects the $M_{k 2}$ and $M_{k 3}$ layers of $M_{k+1}, D_{k+1}$ connects the $M_{k 1}$ and $M_{k 2}$ layers of $M_{k+1}$, $f_{k}^{k+1}\left(C_{k+1}^{\prime \prime}\right)=C_{k}^{\prime \prime}$, and $f_{k}^{k+1}\left(D_{k+1}^{\prime \prime}\right)=D_{k}^{\prime \prime}$. See Figure 3 for the 1-dimensional counterparts of $M_{1}, M_{2}$, and $M_{3}$.

Thus, for each positive integer $i$, we have defined a 2-manifold $M_{i}$ and a three fold covering map $f_{i}^{i+1}: M_{i+1} \rightarrow M_{i}$. Let $\left(M_{i}, f_{i-1}\right)^{i}$ be the corresponding inverse limit sequence and let $M$ be the limit of this sequence. Thus $M$ is the inverse limit of homogeneous spaces where the bonding maps are covering maps.

THEOREM 5. The limit space $M$ is not homogeneous.

Proof. We must show that the conditions of Theorem 4 are satisfied. To say that the point $x$ of $M_{k}$ lies on layer $i$ of $M_{k}$ means that $x$ has a representative 

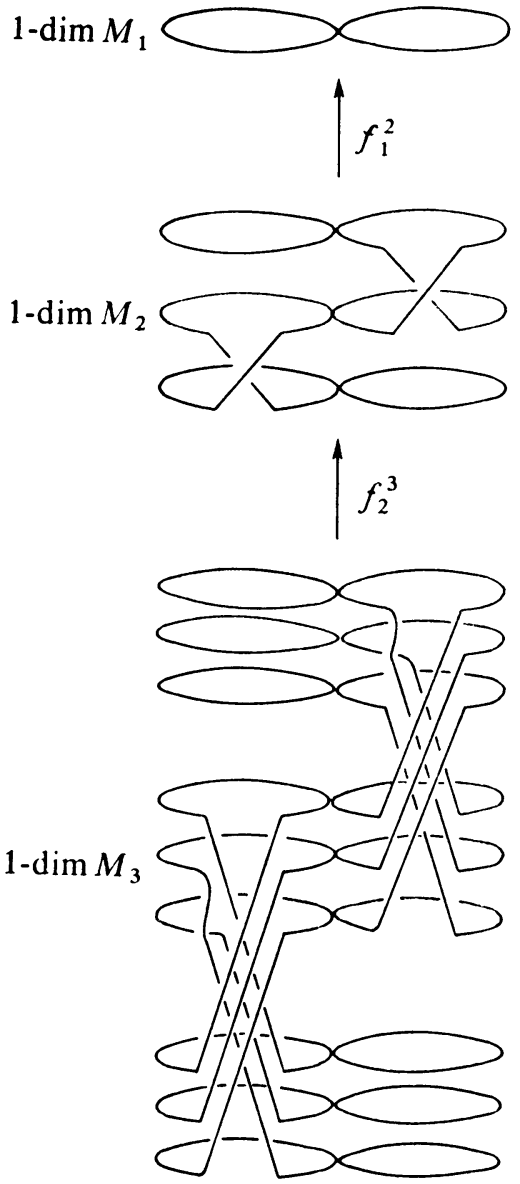

FIGURE 3

$(y, i)$ where $(y, i) \in M_{(k-1) i}$. If $H$ is an $n$-handle, $J$ a closed interval, and $w: J \rightarrow H$ a path, then to say that $w$ goes around $H$ means that $w(J)$ is a simple closed curve that is longitudinal in $H$.

Let $r \in M$ such that for each $k \geqq 1 r_{k}$ lies on layer 1 of $M_{k}$. Let $k>1$ and let $s_{k}$ be a point of $M_{k}$ distinct from $r_{k}$ where $f_{k-1}^{k}\left(s_{k}\right)=r_{k-1}$. Since $r_{k}$ is on layer 1 of $M_{k}$, then $s_{k}$ is either on layer 2 or layer 3. These are completely analogous situations and thus assume $s_{k}$ lies on layer 2 .

Let $p \in H_{k-1}$, the $2^{k-2}$-handle of $M_{k-1}$ that contains the simple closed curve $C_{k-1}^{\prime}$. Let $\alpha_{k-1}: I \rightarrow M \backslash D_{k-1}^{\prime \prime}$ be a loop on $r_{k-1}$ missing $D_{k-1}^{\prime \prime}$ such that (1) from time 0 to $1 / 3 \alpha_{k-1}$ goes from $r_{k-1}=s_{k-1}$ to $p$, (2) $\alpha_{k-1} \mid[1 / 3,2 / 3]$ goes around $H_{k-1}$, and (3) from time $2 / 3$ to $1, \alpha_{k-1}(t)=\alpha_{k-1}(1-t)$. For each $i \geqq k$ lift $\alpha_{k-1}$ to the path $\alpha_{i}: I \rightarrow M_{i}$ with initial point $r_{i}$. In the construction of $M_{k}, C_{(k-1) 1}$ was identified with $C_{(k-1) 1}^{\prime}$ and since $\alpha_{k-1}$ misses $D_{k-1}^{\prime \prime}$ the identification of the $D_{(k-1) i}$ 
have no effect, and thus the loop $\alpha_{k-1}$ lifts to the loop $\alpha_{k}$. Furthermore, for each $i>k$, the loop $\alpha_{k-1}$ lifts to the loop $\alpha_{i}$ and hence the loop $\alpha_{k-1}$ lifts to the loop $\alpha: I \rightarrow M$ on $r$. This is the loop $\alpha$ of Theorem 4 .

We now define the loop $\alpha_{k}^{\prime}$ of Theorem 4. Lift $\alpha_{k-1}$ to the path $\alpha_{k}^{\prime}: I \rightarrow M_{k}$ with initial point $s_{k}$. Since (1) $\alpha_{k} \mid[1 / 3,2 / 3]$ goes around $H_{k-1},(2) s_{k}$ and $\alpha_{k}^{\prime}(1 / 3)$ are on layer 2, and (3) $C_{(k-1) 2}$ is identified with $C_{(k-1) 3}^{\prime}$, then $\alpha_{k}^{\prime}(2 / 3)$ is on layer 3 and so $\alpha^{\prime}(1 / 3) \neq \alpha_{k}^{\prime}(2 / 3)$. From this it follows that $s_{k}=\alpha_{k}^{\prime}(0) \neq \alpha_{k}^{\prime}(1)$. Thus $\alpha_{k}^{\prime}$ is not a loop and since $f_{1}^{k} \alpha_{k}^{\prime}=\alpha_{1}$ all conditions of Theorem 4 have been verified. Hence $M$ is not homogeneous.

Conjecture. Suppose in $\left(M_{i}, f_{i-1}^{i}\right)$ each $M_{i}$ is a closed manifold, each bonding map is a covering map, and $M$ is homogeneous. Then there exists an integer $m$ such that for all $n>m$, the covering map $f_{m}^{n}: M_{n} \rightarrow M_{m}$ is regular. This would be the converse to the theorem of McCord quoted in the introduction.

\section{REFERENCES}

1. M. K. Fort and J. Segal, Local connectedness of inverse limit spaces, Duke Math J. 28 (1961), 253-260.

2. P. J. Hilton and S. Wylie, Homology theory, Cambridge Univ. Press, Cambridge, 1960.

3. M. C. McCord, Inverse limit sequences with covering maps as bounding maps, Trans. Amer. Math. Soc. 114 (1965), 197-209.

4. J. Segal, Homogeneity of inverse limit spaces, Notices Amer. Math. Soc. 5 (1958), 687.

Louisiana State University,

BATON ROLGE, Louisiana 